\title{
Adaptación del Inventario de Resiliencia ante el Suicidio (SRI-25) en adolescentes y jóvenes de Colombia
}

\author{
Fredy Hernán Villalobos-Galvis, ${ }^{1}$ Carolina Arévalo Ojeda ${ }^{1}$ \\ y Fabio Darío Rojas Rivera ${ }^{1}$
}

Forma de citar Villalobos-Galvis FH, Arévalo Ojeda C, Rojas Rivera FD. Adaptación del Inventario de Resiliencia ante el Suicidio (SRI-25) en adolescentes y jóvenes de Colombia. Rev Panam Salud Publica. 2012;31(3):233-9.

RESUMEN Objetivo. Describir las propiedades psicométricas del Suicide Resilience Inventory (SRI25), adaptado al español, en una muestra de adolescentes y jóvenes escolarizados de San Juan de Pasto, Colombia.

Métodos. Se adaptó el SRI-25 al idioma español por medio de una traducción simple. Se contó con 573 participantes, estudiantes de colegios y universidades, de 10 a 25 años de edad.

Resultados. En un análisis factorial exploratorio por componentes principales se encontró una estructura de tres factores que explican el 52,83\% de la varianza. Se realizaron análisis factoriales según el sexo, el nivel educativo y la etapa evolutiva, a partir de los cuales se confirmó que en todos los grupos, los ítems cargan en los mismos factores de la prueba original, a saber: protección interna, estabilidad emocional y protección externa. Se obtuvo un coeficiente alfa de Cronbach de 0,922 para la prueba total. En cuanto a la validez de constructo, el SRI25 presentó correlaciones significativas con medidas de autoestima, apoyo social, solución de problemas, razones para vivir, depresión, desesperanza e ideas suicidas.

Conclusión. Se comprobó que el SRI-25 es un instrumento útil para medir la resiliencia ante el suicidio en adolescentes y jóvenes escolarizados de San Juan de Pasto, Colombia.

Palabras clave Suicidio; resiliencia psicológica; ideación suicida; Colombia.

El suicidio es un problema mental de salud pública, trágico y potencialmente prevenible que se ha convertido en la tercera causa de muerte entre los adolescentes de todo el mundo. En Latinoamérica la tasa de suicidio era de 4,1/100 000 en el 2002, mientras que en Colombia es de 4/100 000 (1), y es de destacar que la mayor frecuencia se observa entre los 20 y los 29 años en los varones, y entre los 10 y los 19 años en las mujeres (2).

\footnotetext{
Universidad de Nariño, Departamento de Psicología, Pasto, Colombia. Enviar correspondencia a Fredy Villalobos-Galvis, fhvillalobos@udenar.edu.co
}

La mayoría de esfuerzos en torno al estudio del suicidio se han orientado a la identificación de factores de riesgo o de protección (prevención primaria); no obstante, no se ha abordado el estudio sistemático de estos mismos factores en personas que ya han presentado ideas o intentos de suicidio (prevención secundaria) (3). Desde esta necesidad, el estudio de la resiliencia se enfoca en aquellas condiciones que permiten que el sujeto se abra a un desarrollo más sano y positivo, en vez de centrar su mirada en circuitos que mantienen este tipo de situaciones desfavorables para él (4).
Desde hace siglos se ha admitido que algunas personas logran superar condiciones severamente adversas y que, inclusive, logran transformarlas en una ventaja o un estímulo para su desarrollo biopsicosocial; sin embargo, el estudio científico de la resiliencia es reciente (5). El origen de este término proviene de la física y alude a la capacidad de un material para volver a su estado inicial después de una deformación producida por una presión externa. El primero en usar el término resiliencia en sentido figurado, fue Bowlby en 1992, quien la definió como "resorte moral, cualidad 
de una persona que no se desanima, que no se deja abatir". En psicología, la resiliencia no se limita a la resistencia, sino que conlleva un significado dinámico que implica que el individuo que pasa por dificultades, se sobrepone $y$ se reconstruye. Alude también a la capacidad humana para sobreponerse a las adversidades y construir sobre ellas, adaptarse, recuperarse y acceder a una vida significativa y productiva (6).

Osman et al. (3) definen la resiliencia ante el suicidio como las habilidades, recursos o competencias percibidas por el sujeto para regular los pensamientos, sentimientos y conductas relacionados con el suicidio. Es decir, la capacidad que tiene el individuo para enfrentar el espectro suicida, una vez que alguna de esas conductas se ha presentado.

El Inventario de Resiliencia ante el Suicidio (Suicide Resilience Inventory, SRI-25), se organiza en tres factores: a) protección interna: alude a los atributos personales y representa las creencias o sentimientos positivos sobre uno mismo y la satisfacción con la propia vida; b) estabilidad emocional: refleja las creencias positivas sobre las habilidades propias para regular las ideas y comportamientos relacionados con el suicidio, cuando se afrontan eventos estresantes difíciles y c) protección externa: representa las habilidades que tienen los individuos para buscar recursos sociales, que pueden ser útiles cuando se afrontan dificultades personales o ideas suicidas (3).

En la primera versión del SRI-25 se encontró una solución de tres factores (68,9\% de varianza explicada) y una fiabilidad de 0,96 para el total de la prueba (3). Rutter, Freedenthal y Osman (7) validaron esta solución de tres factores, y encontraron evidencias de validez concurrente con escalas de desesperanza, ideación suicida y soporte social.

A partir de lo anterior, el presente trabajo buscó determinar las propiedades psicométricas del SRI-25, adaptado al español, en una muestra de adolescentes y jóvenes escolarizados de San Juan de Pasto, Colombia.

\section{MATERIALES Y MÉTODOS}

Se realizó un estudio instrumental, encaminado a la identificación de las propiedades psicométricas y la adaptación cultural de un instrumento de medición psicológica (8).
Participaron 573 estudiantes, muestra representativa del total de estudiantes de secundaria y educación superior de instituciones educativas de la ciudad de San Juan de Pasto, ${ }^{2}$ con un $5 \%$ de error, un $99 \%$ de confianza y una presencia de conductas suicidas estimada en el $30 \%$ (9). Se hizo un muestreo polietápico que implicó inicialmente la estratificación por sector público y privado y luego por nivel educativo (secundario y superior). Posteriormente se procedió a la selección aleatoria de conglomerados, en este caso las instituciones y luego los cursos. Finalmente, sólo se incluyeron en la muestra los estudiantes que aceptaron voluntariamente participar en el estudio. Las edades oscilaron entre 10 y 25 años $(\mathrm{M}=16,76, \mathrm{DE}=4,19)$. El grupo incluyó $49,7 \%$ de varones y $50,3 \%$ de mujeres, estudiantes de primer grado de secundaria hasta $5^{\circ}$ año de universidad. El 49,9\% de los estudiantes pertenecían a instituciones privadas, y el 50,1\% restante, a instituciones públicas.

\section{Instrumentos}

Inventario de Resiliencia ante el Suicidio (SRI-25). Está diseñado para medir los factores que ayudan a preservar de pensamientos y conductas suicidas. El SRI-25 mostró fiabilidades altas $(0,96$ para el total de la prueba $\mathrm{y}>0,9$ para las escalas), así como buenos niveles de validez factorial y validez discriminante para diferenciar los grupos suicidas de los no suicidas (3).

Escala de Apoyo Social (AS). Mide la percepción global del apoyo social, desde la sensación de disponibilidad de apoyo emocional de las personas, a través de las áreas personal, laboral y familiar. La escala presenta buena consistencia interna $(\alpha=0,9)$ y adecuada validez de constructo (10).

Inventario de Solución de Problemas Sociales (SPSI). Evalúa dos dimensiones constructivas o adaptativas de la resolución de problemas y tres dimensiones disfuncionales. Posee un alfa de Cronbach de 0,86 y relaciones significativas con medidas de estrés percibido (11).

\footnotetext{
Dado que no se conoció el número exacto de estudiantes del universo muestral, se tomó la fórmula de tamaño de muestra para poblaciones infinitas, sugerida por Aguilar-Barojas, S. Fórmulas para el cálculo de la muestra en investigaciones en salud. Salud en Tabasco. 2005;11:333-338.
}

Escala de Desesperanza de Beck (BHS). Es un instrumento creado para evaluar un sistema de esquemas cognitivos basados en la expectativa negativa del futuro a mediano y largo plazo. La escala ha presentado buenos datos de fiabilidad y obtiene resultados positivos respecto a su validez, al diferenciar claramente entre los sujetos potencialmente suicidas y los que no lo son (12).

Escala de Depresión del Centro de Estudios Epidemiológicos (CES-D). Permite evaluar síntomas de depresión en la población general, tomando en cuenta afecto negativo, síntomas somáticos y retraso motor, problemas interpersonales y afecto positivo (13). Esta escala tiene una adecuada validez factorial y una alta consistencia interna $(\alpha>0,88)(14)$.

Escala de Autoestima de Rosenberg (AE). Evalúa el conjunto de pensamientos y sentimientos de una persona acerca de su propio valor e importancia, es decir, una actitud positiva o negativa global hacia sí mismo. Presenta una buena consistencia interna $(\alpha=0,87)$ y una confiabilidad test-retest de 0,72 (15).

Inventario de Razones para Vivir (RFL). Valora 5 áreas que pueden proporcionar razones para no cometer suicidio: a) relacionesfamiliares, $b$ ) relacionesconiguales, c) creencias de afrontamiento, d) expectativas frente al futuro y e) autoevaluación positiva. Posee buenos niveles de consistencia interna y de validez de constructo $(16,17)$.

Cuestionario de Ideación Suicida (SIQ). Evalúa la frecuencia de una jerarquía de pensamientos suicidas que van desde los posibles pensamientos de desear no estar vivo, hasta pensamientos serios y específicos de matarse. Posee una adecuada validez factorial y una muy alta consistencia interna $(\alpha=0,97)(18)$.

\section{Procedimiento}

Esta investigación abarcó 7 fases de trabajo, siguiendo el procedimiento de traducción simple. La fase de traducción estuvo a cargo de expertos en lingüística e implicó: a) leer el ítem e identificar su significado en la población estadounidense, b) determinar si dicho ítem tenía un significado similar en español, c) redactar el ítem en español y d) calificar el nivel de dificultad que implicó 
la traducción del ítem. En la fase de consolidación de la versión traducida se generó una nueva versión que implicó la síntesis de las versiones obtenidas de los traductores. La fase de evaluación de jueces comprendió la revisión de la versión traducida consolidada, con el fin de determinar si los reactivos mantenían el sentido que tenían en la prueba original. En la fase cuatro, se definió una versión preliminar que incluía las sugerencias y ajustes que realizaron los jueces. En la fase cinco, el equipo investigador revisó la versión preliminar e hizo ajustes menores que hicieran más fácil su comprensión en el medio cultural en el que sería aplicado el instrumento, buscando la manera más "cotidiana" de expresarlo, para consolidar la versión piloto. La fase de pilotaje evaluó el grado de comprensión que las personas de la ciudad podrían tener de la versión piloto. Para ello se aplicó el instrumento a 25 estudiantes de colegios y universidades de la ciudad. En la última etapa, se consolidó la versión definitiva del instrumento que fue aplicada a la muestra final del estudio.

\section{Consideraciones éticas}

El presente estudio forma parte del proyecto denominado "Validación y Estandarización de Pruebas Psicológicas en Adolescentes Escolarizados de la ciudad de San Juan de Pasto", avalado por el Comité de Ética de la Universidad de Nariño. Considerando que este estudio implicó solamente la aplicación de pruebas psicológicas, el riesgo fue catalogado como "mínimo" (19). Dado que el suicidio es un problema de salud pública del Municipio de Pasto, se contó con la autorización inicial del cuerpo directivo de los colegios y universidades, luego con el consentimiento informado de los padres de familia de los menores de edad $\mathrm{y}$, además, con el asentimiento/ consentimiento informado de los mismos participantes. Adicionalmente, se ofrecieron servicios de atención psicológica a los estudiantes que lo solicitaron. El diligenciamiento de los cuestionarios fue anónimo y se llevó a cabo en los salones de clase en los horarios académicos.

\section{RESULTADOS}

\section{Adaptación y validación lingüísticas}

La aplicación de las 6 primeras etapas del procedimiento permitió la obtención de un instrumento que mantiene los mismos 25 ítems de la versión original y que garantiza su comprensión y pertinencia en adolescentes y jóvenes escolarizados de San Juan de Pasto. En lo que respecta a la fase 3 del procedimiento (evaluación de jueces) es importante aclarar que los expertos no formularon ajustes significativos a los ítems consolidados en la fase 2, considerando que eran claros y mantenían el sentido de la versión original, por lo cual no se tuvo la necesidad de realizar la fase 4 (versión preliminar).

\section{Análisis factorial exploratorio}

Mediante el programa SPSS 16 (SPSS Inc., EE.UU.), se comprobaron los supuestos del análisis factorial, a saber: índice de Kaiser Meyer Olkin cercano a

\section{CUADRO 1. Matriz de patrones factoriales para los ítems del Inventario de Resiliencia ante el Suicidio (SRI-25)}

\begin{tabular}{|c|c|c|c|c|}
\hline & & & Factor & \\
\hline Ítem & Contenido del ítem & 1 & 2 & 3 \\
\hline 25 & Me siento bien conmigo mismo & 0,863 & & \\
\hline 13 & Me siento orgulloso de mí mismo en muchos aspectos & 0,767 & & \\
\hline 5 & Me quiero tal como soy & 0,736 & & \\
\hline 11 & Estoy satisfecho con la mayoría de cosas en mi vida & 0,730 & & \\
\hline 20 & $\begin{array}{l}\text { A pesar de los problemas que enfrente, puedo sentirme bien conmigo } \\
\text { mismo }\end{array}$ & 0,692 & & \\
\hline 19 & Siento que soy una persona emocionalmente fuerte & 0,667 & & \\
\hline 2 & Casi siempre me veo como una persona feliz & 0,654 & & \\
\hline 1 & Hay muchas cosas que me gustan de mí mismo & 0,585 & & \\
\hline 9 & Casi siempre tengo metas que creo que son posibles de alcanzar & 0,417 & & \\
\hline 8 & Aunque me sienta muy mal puedo controlar la idea de suicidarme & & $-0,833$ & \\
\hline 10 & $\begin{array}{l}\text { Aunque me sienta triste o deprimido, puedo controlar las "ganas" de } \\
\text { suicidarme }\end{array}$ & & $-0,818$ & \\
\hline 12 & Si estoy en una situación difícil, puedo evitar pensar en el suicidio & & $-0,786$ & \\
\hline 14 & $\begin{array}{l}\text { Soy capaz de controlar el deseo de hacerme daño o herirme, cuando } \\
\text { alguien me critica }\end{array}$ & & $-0,779$ & \\
\hline 18 & $\begin{array}{l}\text { Cuando me siento solo o aislado de los demás, puedo controlar las ideas } \\
\text { de suicidarme }\end{array}$ & & $-0,773$ & \\
\hline 23 & $\begin{array}{l}\text { Cuando me enfrento a situaciones humillantes o vergonzantes puedo } \\
\text { evitar pensar en el suicidio }\end{array}$ & & $-0,636$ & \\
\hline 24 & Aunque sienta que mi futuro es incierto no pienso en el suicidio & & $-0,578$ & \\
\hline 4 & $\begin{array}{l}\text { Puedo aguantar el dolor que se siente por ser rechazado o criticado, sin } \\
\text { pensar en suicidarme }\end{array}$ & & $-0,532$ & \\
\hline 15 & $\begin{array}{l}\text { Si pensara en suicidarme, puedo pedirle apoyo emocional a mis } \\
\text { familiares o amigos cercanos }\end{array}$ & & & 0,742 \\
\hline 6 & $\begin{array}{l}\text { Si pensara en suicidarme, podría hablar de esto tranquilamente con mis } \\
\text { familiares o amigos }\end{array}$ & & & 0,722 \\
\hline 7 & $\begin{array}{l}\text { Si tengo algún problema, puedo contar con algún familiar o amigo } \\
\text { cercano para que me ayude (por ej., con dinero o alojamiento) }\end{array}$ & & & 0,660 \\
\hline 16 & $\begin{array}{l}\text { Aun cuando mis familiares o amigos cercanos estén disgustados } \\
\text { conmigo, puedo acercarme a ellos para hablar de mis problemas } \\
\text { personales }\end{array}$ & & & 0,650 \\
\hline 17 & $\begin{array}{l}\text { Puedo encontrar a alguien (familiar o amigo) que me ayude a enfrentar la } \\
\text { idea de suicidarme }\end{array}$ & & & 0,648 \\
\hline 22 & $\begin{array}{l}\text { Tengo amigos o familiares a quienes puedo acudir por apoyo emocional, } \\
\text { si llegara a pensar en suicidarme }\end{array}$ & & & 0,648 \\
\hline 3 & $\begin{array}{l}\text { Si yo hablara de suicidarme, mis familiares o amigos hallarían el tiempo } \\
\text { necesario para escucharme }\end{array}$ & & & 0,630 \\
\hline 21 & $\begin{array}{l}\text { Si tengo problemas, puedo pedirle ayuda las personas cercanas a mí, en } \\
\text { vez de intentar suicidarme }\end{array}$ & & & 0,412 \\
\hline
\end{tabular}

Nota: No se registraron las cargas menores de 0,4 .
$1(0,938)$, prueba de esfericidad de Bartlett significativa $\left(\chi^{2}=6573,52 ; \mathrm{gl}=300\right.$; $P<0,0001)$; y comunalidades superiores a $0,2(0,23-0,69)$.

Con el análisis factorial por componentes principales se encontró una solución de 3 factores, con autovalores mayores de 1 , cuya varianza explicada es del $52,83 \%$. Se desarrolló una rotación oblicua por la técnica Oblimin, con normalización de Kaiser (3) (cuadro 1). Por otra parte, se hallaron correlaciones entre los tres factores de la prueba, que oscilaron entre 0,391 (entre los factores 1 y 2) y 0,490 (entre los factores 1 y 3), lo que demostró que aunque estaban correlacionados, cada uno medía aspectos diferentes de la resiliencia ante el suicidio. Estas correlaciones validaron el uso de la rotación oblicua. 
Los tres factores encontrados se nombraron según lo propuesto por Osman et al. (3): a) factor 1, protección interna; b) factor 2, estabilidad emocional y c) factor 3 , protección externa.

El factor 1, "protección interna" (atributos personales), explicó el 35,98\% de la variabilidad de la prueba y estuvo compuesto por ítems caracterizados por la percepción positiva y la aceptación de sí mismo, así como la satisfacción con la vida (véase el cuadro 1 ).

El factor 2, "estabilidad emocional", reflejó las creencias positivas que el individuo tiene acerca de sus habilidades y recursos, para enfrentar ideas o deseos de suicidio cuando vive situaciones estresantes, así como la capacidad de autocontrol de conductas autolíticas (véase el cuadro 1). Este factor explicó el 10,38\% de la varianza.

Finalmente, el factor 3, "protección externa", explicó el 6,48\% de la varianza y representó el soporte social percibido, así como las habilidades necesarias para conseguirlo, en situaciones estresantes o ante la aparición de ideas o deseos de suicidio (véase el cuadro 1).

\section{Validación del modelo factorial según variables sociodemográficas}

Una forma de validar los resultados de un análisis factorial exploratorio es comparar las soluciones factoriales entre subgrupos de la población estudiada, por lo que se realizaron análisis en grupos según sexo, nivel educativo, sector educativo y etapa evolutiva. Al restringir el número de factores a 3, se obtuvo que en todos ellos los ítems se agruparon de acuerdo con la estructura original de la prueba y que la varianza explicada osciló entre 49 y $56 \%$.

\section{Consistencia interna de la prueba total y de las escalas}

Se encontró un buen nivel de consistencia interna ( $\alpha=0,922$ para la prueba total y $0,85<\alpha<0,88$ para las escalas) (cuadro 2). Igualmente, las correlaciones ítem-escala fluctuaron entre 0,39 y 0,74, lo que demuestra una alta interrelación de los reactivos que componen la prueba.

\section{Validez de constructo}

De acuerdo con la bibliografía sobre conductas suicidas y variables de riesgo y protección, cabría esperar que la resi- liencia ante al suicidio presentara una correlación positiva con autoestima (20, 21), apoyo social, solución de problemas y razones para vivir (22), así como relaciones negativas con desesperanza (23), depresión (24) e ideas suicidas (25).

De acuerdo con lo anterior, el SRI25 demostró validez de constructo, al presentar correlaciones estadísticamente significativas $(P<0,001)$, y en la dirección predicha, con todas las variables consideradas en el estudio (cuadro 3). Se destacaron las correlaciones del SRI-25 con medidas de autoestima, solución de problemas, razones para vivir y depresión.

\section{Diferencias en los puntajes \\ directos de acuerdo con variables sociodemográficas}

La prueba Kruskal-Wallis, aplicada tanto a la prueba total como a las escalas, demostró diferencias entre las puntuaciones según el nivel educativo. Esto implica que a la hora de evaluar los resultados del inventario, se deben considerar los estadísticos de distribución para cada grupo (cuadro 4). Así, para poder interpretar las puntuaciones de una persona en el inventario, es obligatorio considerar su nivel de escolaridad, pues de él dependen los parámetros de comparación que se deben aplicar. Es importante aclarar que los niveles de resiliencia se calcularon sobre la base de los cuartiles. Por ejemplo, si una persona presenta una calificación directa que se ubica en el nivel mínimo, su grado de resiliencia no superaría al $25 \%$ de las puntuaciones halladas en la muestra de referencia, es decir que está por debajo de los niveles medios de presentación de esta variable. Por el contrario, si una persona presenta calificaciones que se ubican en el nivel "muy alto", se puede asumir que tiene muy buenos recursos de resiliencia frente al suicidio ya que supera, como mínimo, al 75\% de la muestra de estudio. A partir de esto, se asume que a medida que se incrementa la puntuación en el SRI-25, el nivel de resiliencia ante el suicidio también aumenta.

\section{DISCUSIÓN}

El presente estudio identificó las propiedades psicométricas del Inventario de Resiliencia ante el Suicidio (SRI-25) (3), adaptado al español, en una muestra de adolescentes y jóvenes escolarizados de la ciudad de San Juan de Pasto, Colombia. Se demostró que el SRI-25 tiene una estructura de tres factores que coinciden con la propuesta original, altos niveles de consistencia interna (en la prueba total y sus escalas) e importantes relaciones con medidas de autoestima, apoyo social, solución de problemas, razones para vivir, depresión, ideas suicidas y desesperanza. Además, existen diferencias en las puntuaciones total y por escalas, de acuerdo con el nivel educativo.

CUADRO 2. Valores de la consistencia interna de la prueba total y de las escalas del Inventario de Resiliencia ante el Suicidio (SRI-25)

\begin{tabular}{lcccc}
\hline & $\begin{array}{c}\text { Mínima } \\
\text { Alfa de } \\
\text { Cronbach }\end{array}$ & $\begin{array}{c}\text { Máxima } \\
\text { correlación } \\
\text { ítem - prueba }\end{array}$ & $\begin{array}{c}\text { correlación } \\
\text { ítem - prueba }\end{array}$ & $\begin{array}{c}\text { Número } \\
\text { de ítems }\end{array}$ \\
\hline Protección interna & 0,867 & 0,394 & 0,736 & 9 \\
Estabilidad emocional & 0,884 & 0,478 & 0,729 & 8 \\
Protección externa & 0,852 & 0,472 & 0,688 & 8 \\
Total & 0,922 & 0,395 & 0,671 & 25 \\
\hline
\end{tabular}

CUADRO 3. Correlaciones del Inventario de Resiliencia ante el Suicidio (SRI-25) con medidas de otras variables relacionadas teóricamente con la resiliencia

\begin{tabular}{lccccccc}
\hline & AE & AS & SPSI & BHS & CES-D & SIQ & RFL \\
\hline Protección interna & 0,700 & 0,407 & 0,518 & $-0,391$ & $-0,527$ & $-0,488$ & 0,496 \\
Estabilidad emocional & 0,490 & 0,292 & 0,387 & $-0,329$ & $-0,382$ & $-0,305$ & 0,288 \\
Protección externa & 0,571 & 0,534 & 0,426 & $-0,353$ & $-0,387$ & $-0,318$ & 0,465 \\
SRI total & 0,694 & 0,472 & 0,514 & $-0,432$ & $-0,512$ & $-0,422$ & 0,497 \\
\hline
\end{tabular}

AE, Escala de Autoestima de Rosenberg; AS, Escala de Apoyo Social; SPSI, Inventario de Solución de Problemas; BHS, Escala de Desesperanza de Beck; CES-D, Escala de Depresión del Centro de Estudios Epidemiológicos; SIQ, Cuestionario de Ideación Suicida; RFL, Inventario de Razones para Vivir. 
CUADRO 4. Distribución de las puntuaciones de la prueba total y las escalas del Inventario de Resiliencia ante el Suicidio (SRI-25), según el nivel educativo de la muestra

\begin{tabular}{llrrrr}
\hline Escolaridad & \multicolumn{1}{c}{ Nivel de resiliencia } & Total & $\begin{array}{r}\text { Protección } \\
\text { interna }\end{array}$ & $\begin{array}{r}\text { Estabilidad } \\
\text { emocional }\end{array}$ & $\begin{array}{c}\text { Protección } \\
\text { externa }\end{array}$ \\
\hline Bachilleres & Mínimo & $0-51$ & $0-19$ & $0-16$ & $0-15$ \\
& Moderado & $52-57$ & $20-22$ & $17-19$ & $16-18$ \\
& Alto & $58-66$ & $23-24$ & $20-22$ & $19-21$ \\
& Muy alto & $67-75$ & $25-27$ & $23-24$ & $21-24$ \\
& Media & 53,034 & 20,012 & 16,996 & 16,072 \\
& Desviación estándar & 12,728 & 4,736 & 5,440 & 5,093 \\
& Mínimo & $0-54$ & $0-20$ & $0-18$ & $0-16$ \\
& Moderado & $55-63$ & $21-23$ & $19-22$ & $17-19$ \\
& Alto & $64-68$ & $24-25$ & 23 & $20-22$ \\
& Muy alto & $69-75$ & $26-27$ & 24 & $23-24$ \\
& Media & 57,050 & 20,972 & 18,968 & 17,109 \\
& Desviación estándar & 11,719 & 4,417 & 4,952 & 4,863 \\
\hline
\end{tabular}

Ante la necesidad de contar con instrumentos que permitan la evaluación de un mismo fenómeno en estudios interculturales (26), en el presente trabajo se realizó la adaptación cultural del SRI-25. Al finalizar el proceso de adaptación se demostró que los 25 ítems de la prueba eran comprensibles para la muestra de estudio y, en consecuencia, no se realizaron cambios a la prueba ni a las opciones de respuesta.

Con el análisis factorial se comprobó que el SRI-25 adaptado al español mantuvo los 3 factores propuestos por Osman et al. (3) y confirmados por Rutter et al. (7). En tales estudios la varianza explicada se mantuvo cercana al $68,1 \%$, mientras que en este trabajo fue del $52 \%$.

El primer factor encontrado fue la protección interna, definida como los atributos personales que los sujetos tienen para sentirse satisfechos con sus acciones y su manera de vivir, que ayudan a contrarrestar las conductas autodestructivas (3). Uno de tales atributos es la autoestima, que ha sido ampliamente documentada como un factor de protección ante la conducta suicida $(20,27)$.

En segundo lugar, se encontró el factor estabilidad emocional, entendida como los recursos internos de un sujeto y el modo en que los regula para hacerle frente a una conducta suicida de manera adaptativa (3). Es necesario resaltar la gran importancia de las habilidades de solución de problemas que le permiten al individuo identificar o descubrir soluciones efectivas o adaptativas a problemas específicos y regular y aplicar sus recursos en el afrontamiento de conflictos o situaciones desequilibrantes que se le presenten en la vida cotidiana. Tales capacidades son cruciales durante la adolescencia y la adultez temprana, puesto que las estrategias ineficaces son un factor de riesgo importante en la conducta suicida (22).

Finalmente, se halló el factor protección externa, definida como los recursos sociales que se encuentran en el ambiente y que ayudan al individuo a enfrentar las ideas de suicidio (3). Este hallazgo confirmó que el soporte social tiene un papel muy importante en la prevención tanto de la ideación como de la conducta suicida en niños, adolescentes $\mathrm{y}$ adultos $(5,22,28)$.

Se encontró un alto nivel de confiabilidad del SRI-25, situación similar a lo reportado por Osman et al. (3) y Rutter et al. (7), que permite afirmar que la prueba tiende a presentar altos coeficientes de consistencia interna.

Por su parte, los resultados de validez concurrente guardaron coherencia con la teoría sobre resiliencia frente al suicidio, pues se presentaron correlaciones positivas con medidas de autoestima, apoyo social, solución de problemas sociales y razones para vivir; y negativas con medidas de depresión, desesperanza e ideación suicida.

En el caso de la autoestima, se ha encontrado que los adolescentes no suicidas tienen un autoconcepto estable relacionado con una autoestima alta, y bajos niveles de depresión y soledad (20). Por el contrario, la baja autoestima se correlaciona con ideas suicidas y suicidio, así como con desesperanza y depresión (18, 21, 27).

El soporte social se ha documentado como un factor protector frente a la conducta suicida en jóvenes (22) ya que permite al sujeto movilizar una mayor variedad de formas de ayuda para hacer frente a las dificultades de la vida (28). Además, "existe una relación directa entre la presencia de un apoyo social eficaz y el equilibrio psicológico de una persona" (5).

Por otra parte, la percepción de soporte social tiene gran importancia como predictor de bajos niveles de ideación suicida, y las personas que evidencian comportamientos resilientes tienden a percibir que tienen un soporte social adecuado $(5,21,25)$.

De igual forma, la solución de problemas también ha sido asociada a la resiliencia. Las personas con comportamientos resilientes manifiestan buenas habilidades para la solución de problemas (5), y ésta es la variable que presenta mayor valor protector frente a la ideación suicida (29).

En cuanto a la depresión, se ha demostrado que los estudiantes que presentaban un historial de ideación suicida evidenciaban más depresión, desesperanza y pensamientos suicidas (24). La depresión está ampliamente relacionada con la ideación suicida, puesto que ésta ha sido encontrada en un gran número de conductas suicidas y ha sido señalada como un predictor significativo, tanto de la tentativa de suicidio como del suicidio consumado (30). En este sentido, las personas que evidencian comportamientos resilientes manifiestan menores índices de depresión y son capaces de tomar distancia de sus problemas y verlos desde una perspectiva más positiva, e incluso bromean al respecto (31).

En otros estudios se ha demostrado la relación entre el suicidio y la desesperanza (23) al ser ésta predictora de la ideación suicida que, cuando se asocia con la depresión, genera tendencias y conductas suicidas más graves en los adolescentes. En la misma línea, la desesperanza es la variable cognitiva más consistentemente asociada a la ideación suicida, así como a los intentos de suicidio y al suicidio consumado (21).

Por el contrario, el contar con un sentido de vida es un elemento que permite sobrellevar las dificultades y le facilita a las personas tener una visión más amplia de sus posibilidades y de la situación en la que viven, y permite que se sientan capaces de proyectarse al futuro, una característica muy importante de la resiliencia $(6,32)$.

La diferencia en los puntajes directos de bachilleres y universitarios permite suponer que la educación es una condición que favorece el desarrollo de la resiliencia (4). Así pues, las personas 
que cuenten con un nivel de educación superior pueden ver aumentadas sus posibilidades de ser resilientes ante diferentes eventos.

La identificación de niveles de resiliencia ante el suicidio permite al profesional que administra la prueba determinar cuándo una persona tiene valores altos, medios o bajos de la variable, en relación con la respuesta "típica" de la muestra de estudio.

Este estudio presenta algunas limitaciones. Un primer aspecto es que se llevó a cabo con estudiantes voluntarios, por lo cual se encontraron varios casos de no respuesta, es decir, de personas que no quisieron participar en el estudio o cuyos padres no dieron la autorización. Desafortunadamente, no se llevó a cabo un registro de tales casos, ni se implementó una estrategia estadística para el manejo del sesgo por no respuesta.

Dado el carácter transversal del estudio, sólo se hizo una aplicación del SRI-25, esto impidió calcular los niveles de confiabilidad test-retest. Es importante que se realicen estudios en este sentido, con seguimientos a 1, 3, 6 y 12 meses, pues servirían de referencia para sugerir el uso posterior del SRI-25 como un indicador de avance terapéutico de intervenciones en casos de conductas suicidas no letales.

Por otro lado, se sugiere realizar estudios de validez clínica de la prueba, en el sentido de corroborar si la presencia de resiliencia ante el suicidio, en personas que han presentado un intento de suicidio, es efectivamente un factor que disminuye la probabilidad de un nuevo episodio suicida.

El presente estudio se llevó a cabo con población de fácil acceso dada su vinculación con una institución educativa, y la educación representa un dispositivo social de inclusión. Sería de gran importancia corroborar si las propiedades psicométricas del instrumento se mantienen en otros grupos con menor inclusión social, como personas no escolarizadas o con vida de calle ("sin hogar"), o personas de otros niveles de desarrollo tales como adultos y adultos mayores.

Finalmente, esta investigación se llevó a cabo en una ciudad mediana (aproximadamente 400000 habitantes), en la que se ha destacado el fenómeno del suicidio como un problema de salud pública. Lo anterior puede generar un sesgo positivo, ya que las personas pueden reconocer y expresar con más facilidad la presencia de conductas suicidas y de variables relacionadas con ellas. Se sugiere llevar a cabo estudios en otras ciudades que permitan evaluar la validez de dicho posible sesgo.

A partir de las particularidades del concepto de resiliencia ante el suicidio y de los resultados de esta investigación, se sugiere el uso del SRI-25 en los casos en los que se haya detectado la presencia de alguna conducta suicida (ideas, planes o intentos) o de factores de riesgo para ella, como depresión, desesperanza y eventos vitales estresantes (11). Lo anterior permitirá orientar los respectivos procesos de prevención secundaria o terciaria, haciendo énfasis en aquellas personas que demuestren bajas puntuaciones en la prueba total o en alguna de las escalas y orientando la intervención de manera específica al aspecto más pertinente.

Finalmente, se puede concluir que el SRI-25 es una prueba adaptada a las condiciones culturales de los adolescentes y jóvenes escolarizados de San Juan de Pasto, que cumple con los criterios de validez factorial, consistencia interna y validez concurrente, por lo que se sugiere su uso en personas con características similares a las de quienes participaron en esta investigación.

\section{REFERENCIAS}

1. Instituto Nacional de Medicina Legal y Ciencias Forenses. Forensis. 2008. Disponible en http://www.medicinalegal.gov.co/index. php?option=com_wrapper\&Itemid $=323$ Acceso el 19 de enero de 2010.

2. Cendales R, Vanegas C, Fierro M, Córdoba $\mathrm{R}$, Olarte A. Tendencias del suicidio en Colombia, 1985-2002. Rev Panam Salud Publica. 2007;22(4):231-8.

3. Osman A, Gutiérrez P, Muehlenkamp J, DixRicharson F, Barrios F, Kopper B. Suicide Resilience Inventory-25: development and preliminary psychometric properties. Psychol Rep. 2004;94:1349-60.

4. Kotliarenco M, Cáceres I, Álvarez C. Resiliencia: construyendo en adversidad. Santiago de Chile: CEANIM; 1996.

5. Vanistendael S, Lecomte J. La felicidad es posible. Despertar en niños maltratados la confianza en sí mismos: construir la resiliencia. Barcelona: Gedisa; 2002.

6. Manciaux M, Vanistendael F, Lecomte J, Cyrulnik B. La resiliencia: estado de la cuestión. En: Manciaux M (comp.) La resiliencia. Resistir y rehacerse. Barcelona: Gedisa; 2003. Pp. 17-27.

7. Rutter P, Freedenthal S, Osman A. Assessing Protection from Suicidal Risk: Psychometric Properties of The Suicide Resiliency Inventory. Death Stud. 2008;32:142-53.
8. Montero I, León O. A guide for naming research studies in Psychology. Int J Clin Health Psychol. 2007;3:847-62.

9. Villalobos-Galvis, F. Situación de la conducta suicida en estudiantes de colegios y universidades de San Juan de Pasto. Salud Ment. (Mex). 2009;32:165-71.

10. Matud P, Carballeira M, López M, Marrero R, Ibáñez I. Apoyo social y salud: Un análisis de género. Salud Ment. (Mex). 2002;25:32-7.

11. Villalobos-Galvis, F. Ideación suicida en jóvenes: Formulación y validación del Modelo Integrador Explicativo en estudiantes de educación secundaria y superior [tesis doctoral]. Granada: Universidad de Granada; 2009.

12. Beck A, Steer R. Beck Hopelessness Scale. Manual. New York: The Psychological Corporation; 1988 .

13. Pettit J, Lewinsohn P, Seeley J, Roberts R, Hibbard J, Hurtado A. Association between the Center for Epidemiologic Studies Depression Scale (CES-D) and mortality in a community sample: An artifact of the somatic complaints factor. Int J Clin Health Psychol. 2008;383-97.

14. Villalobos-Galvis, F. Estructura factorial y propiedades psicométricas de una escala de depresión en universitarios de Colombia. Rev Panam Salud Publica. 2010;27:110-6.
15. Vázquez A, Jiménez R, Vásquez R. Escala de autoestima de Rosenberg: fiabilidad y validez en población clínica española. Apunt Psicol. 2004;22:247-55.

16. Osman A, Downs W, Kopper B, Barrios F, Baker M, Osman J, Besset T, Linehan M. The Reasons for living inventory for adolescents (RFL-A): Development and psychometric properties. J Clin Psychol. 1998;54: 1063-78.

17. Moreno, A. Propiedades psicométricas del Inventario de Razones para Vivir adaptado al español, versión para adolescentes, con estudiantes de San Juan de Pasto [Trabajo de grado en psicología]. Pasto: Universidad de Nariño; 2008.

18. Villalobos F, Sevillano F. Caracterización psicométrica de una versión reducida del Cuestionario de Ideación Suicida SIQ-HS (Reynolds y Mazza, 1987), adaptada al español, en una muestra de estudiantes de la ciudad de San Juan de Pasto. Universidad y Salud. 2008;8:32-44.

19. Ministerio de Salud de Colombia. Resolución 8430 , por la cual se establecen las normas científicas, técnicas y administrativas para la investigación en salud; 1993.

20. Grøholt B, Ekeberg Ø; Wichstrøm L, Haldorsen T. Suicidal and Nonsuicidal Adolescents: Different Factors Contribute to Self- 
Esteem. Suicide Life Threat Behav. 2005;35: 525-35.

21. Chioqueta A, Stiles T. The Relationship Between Psychological Buffers, Hopelessness, and Suicidal Ideation. Identification of Protective Factors. Crisis. 2007;28: 67-73.

22. Eskin M, Ertekin K, Dereboy C, Demirkiran F. Risk Factors for and Protective Factors Against Adolescent Suicidal Behavior in Turkey. Crisis. 2007;28:131-9.

23. Beevers C, Miller I. Perfectionism, Cognitive Bias, and Hopelessness as Prospective Predictors of Suicidal Ideation. Suicide Life Threat Behav. 2004;34(2):126-37.

24. Garnefski H, Diekstra R, De Heus P. A population-based survey of the characteristics of high school students with and with a history of suicidal behavior. Acta Psychiatr Scand. 1992;86:189-96.

25. Rutter P, Behrendt A. Adolescent suicide risk: four psychosocial factors. Adolescence. 2004;39(154):295-302.

26. Hambleton R, Patsula L. Adapting tests for use in multiple languages and cultures. Soc Indic Res. 1998;45:153-71.

27. Wild L, Flisher A, Lombard C. Suicidal ideation and attempts in adolescents: associations with depression and six domains of self-esteem. J Adolesc. 2004;27:611-24.

28. Cheng S, Chan A. Multiple Pathways from Stress to Suicidality and the Protective Effect of Social Support in Hong Kong Adolescents. Suicide Life Threat Behav. 2007;37:187-96.

29. Cabrera R, Castillo L. Validación de un modelo de factores protectores frente a la idea- ción suicida en adolescentes, estudiantes de bachillerato, de la ciudad de San Juan de Pasto [Trabajo de grado en psicología]. Pasto: Universidad de Nariño; 2007.

30. Rudd M. An integrative model of Suicidal Ideation. Suicide Life Threat Behav. 1990;20: 16-30.

31. Kotliarenco M, Cáceres I, Fontecilla M. Estado del arte en resiliencia. Chile: CEANIM; 1997.

32. Cyrulnik B. El murmullo de los fantasmas. Volver a la vida después de un trauma. Barcelona: Gedisa; 2003.

Manuscrito recibido el 31 de marzo de 2011. Aceptado para publicación, tras revisión, el 15 de agosto de 2011.

ABSTRACT Objective. Describe the psychometric properties of the Suicide Resilience Inventory (SRI-25), adapted to Spanish, in a student sample of adolescents and young adults from San Juan de Pasto, Colombia.

Adapting the Suicide Resilience Inventory (SRI-25) for adolescents and young adults in Colombia

Methods. The SRI-25 was adapted to the Spanish language by means of a simple translation. This involved the participation of 573 schoolchildren and college/ university students between 10 and 25 years of age.

Results. In an exploratory factorial analysis by principal components, a structure of three factors was found to explain $52.83 \%$ of the variance. Factorial analysis was carried out by sex, educational level, and developmental stage. On this basis, it was confirmed that in all the groups, the items are weighted on the same factors as in the original test: namely, internal protection, emotional stability, and external protection. A Cronbach's alpha coefficient of 0.922 was obtained for the total test. Concerning the validity of the construct, the SRI-25 presented significant correlations with measurements of self-esteem, social support, problem-solving skills, reasons for living, depression, despair, and suicidal ideas.

Conclusions. It was proved that the SRI-25 is a useful instrument for measuring resilience to suicide among adolescents and young people pursuing their education in San Juan de Pasto, Colombia.

Key words Suicide; resilience, psychological; suicidal ideation; Colombia. 